\title{
Descompresión suboccipital percutánea como alternativa en el tratamiento del dolor secundario a la neuralgia del nervio occipital: serie de casos
} Suboccipital decompression as an alternative in the treatment of pain secundary to occipital neuralgia: a case series

\author{
G. I. Herrejón Galaviz',
}

y J. C. Torres Huerta ${ }^{1,3}$

${ }^{1}$ Algología Intervencionista. Centro Médico Nacional "20 de noviembre" ISSSTE. Ciudad de México. ${ }^{2}$ Algología. Hospital General de México. Ciudad de México. ${ }^{3} \mathrm{FIPP}$ World Institute of Pain. Ciudad de México

\section{ABSTRACT}

Introduction: Occipital neuralgia is an entity with welldefined components, but occasionally underdiagnosed that tends to maintain insidious behavior and generate some resistance to medical treatment, maintaining a good response to interventional management with a minimum rate of associated complications and pain relief. Satisfactory and prolonged.

Case description: We present 5 cases of patients with defined criteria for occipital neuralgia by (IHS) which were managed by suboccipital decompression, evaluated by the analog numerical scale and analogous verbal scale over a period of 90 days. The procedures were performed without complications or adverse events. The result was a satisfactory relief for the patient of chronic pain, with adequate specificity of the site of the condition; as well as improvement in the quality of life, mood, medication consumption and patient satisfaction.

Conclusions: We consider that the use of decompression of the major occipital nerve via suboccipital route can be taken as an alternative in patients with occipital neuralgia, with a high probability of obtaining an analgesic benefit and a low probability of presenting some adverse effect or complication.

Key words: Arnold's neuralgia, occipital neuralgia, suboccipital neuralgia, occipital nerve.

\section{RESUMEN}

Introducción: La neuralgia occipital es una entidad con componentes bien definidos, pero ocasionalmente subdiagnosticada, que tiende a mantener un comportamiento insidioso y generar cierta resistencia al tratamiento médico, manteniendo una buena respuesta al manejo intervencionista con un mínimo índice de complicaciones asociadas y un alivio del dolor satisfactorio y prolongado.

Descripción del caso: Presentamos 5 casos de pacientes con criterios definidos para neuralgia occipital por la (IHS), los cuales fueron manejados mediante descompresión suboccipital percutánea, evaluados por la escala numérica análoga y escala verbal análoga durante un periodo de $\mathbf{9 0}$ días. Los procedimientos se realizaron sin complicaciones ni eventos adversos. El resultado fue un alivio satisfactorio para el paciente del dolor crónico, con una adecuada especificidad del sitio del padecimiento, así como mejoría en la calidad de vida, el estado de ánimo, el consumo de medicamentos y la satisfacción de la paciente.

Conclusiones: Consideramos que el uso de la descompresión percutánea del nervio occipital mayor vía suboccipital puede ser tomada como una alternativa en pacientes con neuralgia occipital, con una alta probabilidad de obtener un beneficio analgésico y una baja probabilidad de presentar algún efecto adverso o complicación.

Palabras clave: Neuralgia de Arnold, neuralgia occipital, descompresión suboccipital percutánea, nervio occipital.

Recibido: 29-01-2020

Aceptado: 06-05-2020

Correspondencia: Juan Carlos Torres Huerta drtorres@dolortech.mx 


\section{INTRODUCCIÓN}

De acuerdo con la definición de la sociedad internacional de cefaleas (IHS), la neuralgia occipital (también conocida como neuralgia de Arnold) se refiere a un dolor asociado de tipo punzante paroxístico en el territorio de distribución del nervio occipital mayor o el menor, ocasionalmente asociado a disestesias del área afectada [1]. El dolor puede iniciar sobre la región suboccipital irradiado hacia el vértice occipital, región cervical superior e incluso dolor referido retro ocular [2].

La causa más común de la neuralgia occipital es la compresión sobre los nervios occipitales mayor, menor o el tercero en cualquier parte de su trayecto. La teoría fisiopatológica más aceptada sugiere un proceso inflamatorio del nervio occipital debido a atrapamiento o irritación de este en un alguna parte de su trayecto, incluyendo desde su origen en $\mathrm{C} 2$, entre el axis y el atlas, entre el músculo oblicuo menor y el músculo semiespinoso de la cabeza, así como al atravesar la aponeurosis del músculo trapecio, provocando edema e isquemia neural [3-5]. Sin embargo, se han sugerido otras teorías como la que se describe en el estudio de Longo y cols., que nos ayudaría a explicar la eficacia del bloqueo neural en la articulación inflamada y en las zonas normalmente afectadas por procesos degenerativos osteoartrósicos y artríticos. En este modelo animal con monoartritis se muestra que hay una hipersensibilidad persistente al dolor, acompañada de un brote anormal de fibras simpáticas en la piel adyacente a la articulación inflamada. Dicho crecimiento anormal de estas fibras se acompañó por niveles elevados de mNGF (murine Nerve Growth Factor), pero no de su precursor el proNGF, logrando una modulación en los niveles del mNGF, un punto interesante en el tratamiento de la artritis inflamatoria. Observaron que la supresión en la función de las fibras simpáticas lleva a una mejoría en el cuadro doloroso. Estos datos refuerzan el concepto de la existencia de un componente neuropático en la artritis y de que las fibras simpáticas juegan un papel en la génesis del dolor asociado con la inflamación [6].

El tratamiento médico se enfoca en reducir la tensión muscular, y como consecuencia liberar la compresión nerviosa. Para el alivio del dolor occipital, la terapia farmacológica puede ser a base de antidepresivos tricíclicos, antiepilépticos, relajantes musculares, opioides y antinflamatorios no esteroideos (7).

Somos conscientes de la dificultad de homogeneizar los tratamientos previos que han seguido los pacientes antes de llegar a la Unidad del Dolor, pero para futuros estudios conviene elaborar un algoritmo de indicaciones terapéuticas previas al bloqueo. Patwardhan y cols. mencionan en su estudio que una situación que debe considerar es el impacto mecánico-patológico en la columna cervical de una mala alineación en el plano sagital de la misma, la cual puede estar asociada a trastornos, tales como cefalea cervicogénica o dolor cervical. Los resultados en su trabajo consideran que nos pueden ayudar a la toma de decisiones clínicas y, por lo tanto, a la toma de decisiones de tratamiento [8].

Otra de las alternativas es el bloqueo diagnósticoterapéutico de los nervios occipitales mayor y menor en su sitio de inserción sobre la protuberancia occipital, produciendo mejoría del cuadro doloroso. Sin embargo, se reporta una eficacia limitada en relación con la duración del efecto, posiblemente debido a la trayectoria de nervio y su paso por los diferentes músculos que pueden causar atrapamiento. Güvençer y cols. muestran la relación entre el nervio occipital mayor (GON) y los puntos de referencia óseos externos para realizar los bloqueos nerviosos de forma segura, y define los puntos donde el GON perfora la musculatura cervical, identificando lugares donde puede quedar atrapado [9].

Existen estudios donde se demuestra que la descompresión suboccipital percutánea como nueva técnica y la descripción de esta, lo que puede ser una opción terapéutica para el tratamiento de la neuralgia occipital (10-12].

Motivo por el cual este trabajo describe nuestra experiencia en el manejo de los pacientes con neuralgia occipital con la descompresión del nervio occipital mayor mediante un abordaje suboccipital percutáneo para el alivio del dolor.

\section{DESCRIPCIÓN DEL ESTUDIO}

Presentamos a continuación el reporte de cinco casos valorados en el servicio de Clínica del Dolor del Centro Médico Nacional "20 de noviembre" del Instituto de Seguridad y Servicios Sociales de los trabajadores del Estado (ISSSTE), con criterios clínicos de neuralgia occipital de acuerdo con la IHS, a los cuales se les realizó la descompresión suboccipital.

\section{Caso 1}

Paciente femenina de 43 años, con comorbilidades asociadas controladas tales como hipertensión arterial y dislipidemia. Refiere dolor de dos meses de evolución a nivel cervical unilateral izquierda, con irradiación a la región occipital ipsilateral, de características punzante, ardoroso de manera continua, que se acompaña de paroxismos intermitentes (descargas eléctricas) sobre el trayecto del nervio occipital mayor, refiriendo una intensidad del dolor severa (8/10) mediante la Escala Numérica Análoga (ENA) y la Escala Visual Análoga (EVA). Tratamiento conservador mediante antinflamatorios no esteroideos, relajantes musculares centrales y terapia de rehabilitación, sin presentar un adecuado alivio del dolor. Debido a lo anterior, se decidió realizar una infiltración diagnóstica con anestésico local del nervio occipital mayor, en su salida del hueso occipital, trazando una línea imaginaria entre la apófisis mastoides y la tuberosidad occipital externa, se administraron $3 \mathrm{ml}$ (60 mg) de lidocaína al $2 \%$ y se observó una mejoría del cuadro doloroso de manera inmediata con una duración aproximada de seis horas, por lo que se consideró candidata para realizar una descompresión suboccipital izquierda.

Previo a la realización del procedimiento, se explicaron a la paciente los posibles riesgos, las posibles complicaciones, aceptando la realización del procedimiento y se firmó el consentimiento informado. Se realizó procedimiento en sala de fluoroscopia mediante un 


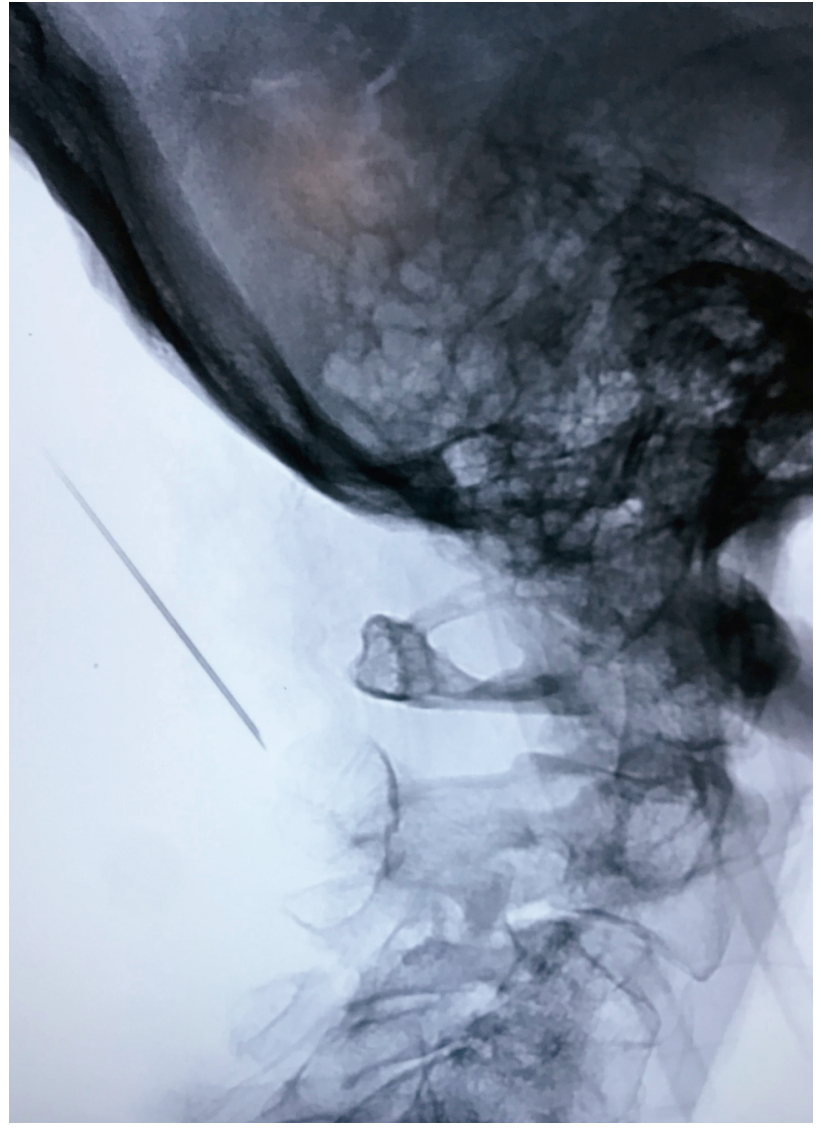

Fig. 1.

arco en $\mathrm{C}$, se colocó a la paciente en posición decúbito prono y con flexión cervical, con monitorización Tipo 1 , se administró oxígeno suplementario por catéter nasal a un flujo de $3 \mathrm{l} / \mathrm{min}$ y sedación intravenosa con fentanilo (1 $\mathrm{mcg} / \mathrm{kg}$ ) y midazolam $[0,05 \mathrm{mcg} / \mathrm{kg}$ ) y anestesia local.

La técnica que se utilizó fue descrita por Racz y cols. (12), se localizaron mediante guía fluoroscópica a través de un arco $\mathrm{C}$ y en una posición anteroposterior las vértebras cervicales 1 y 2 , posteriormente se rotó el equipo de arco en $\mathrm{C}$ a $90^{\circ}$ para observar una proyección lateral, localizando las apófisis espinosas de las vértebras cervicales 1 y 2 (C-1 y C-2). Se eligió el punto de abordaje, localizado a 2 centímetros lateral izquierda de la protuberancia occipital mayor en la línea nucal superior, se infiltró piel cabelluda y tejido celular subcutáneo con lidocaína simple al $2 \%$. Posteriormente se introdujo una aguja espinal BD tipo Quincke 22 G $88 \mathrm{~mm}$, en sentido cefalocaudal y lateromedial; de manera cuidadosa se avanzó a través de las capas fasciales musculares para evitar una lesión nerviosa o vascular (entre las que se encuentran la raíz nerviosa de C-1 o nervio suboccipital, raíz nerviosa de C-2 o tercer nervio occipital, el plexo cervical, la arteria cervical ascedente, la arteria o vena cervical profunda, y la arteria o vena vertebral] hasta hacer contacto con apófisis espinosa de C-2 (Figura 1); se retiró 2 mm y

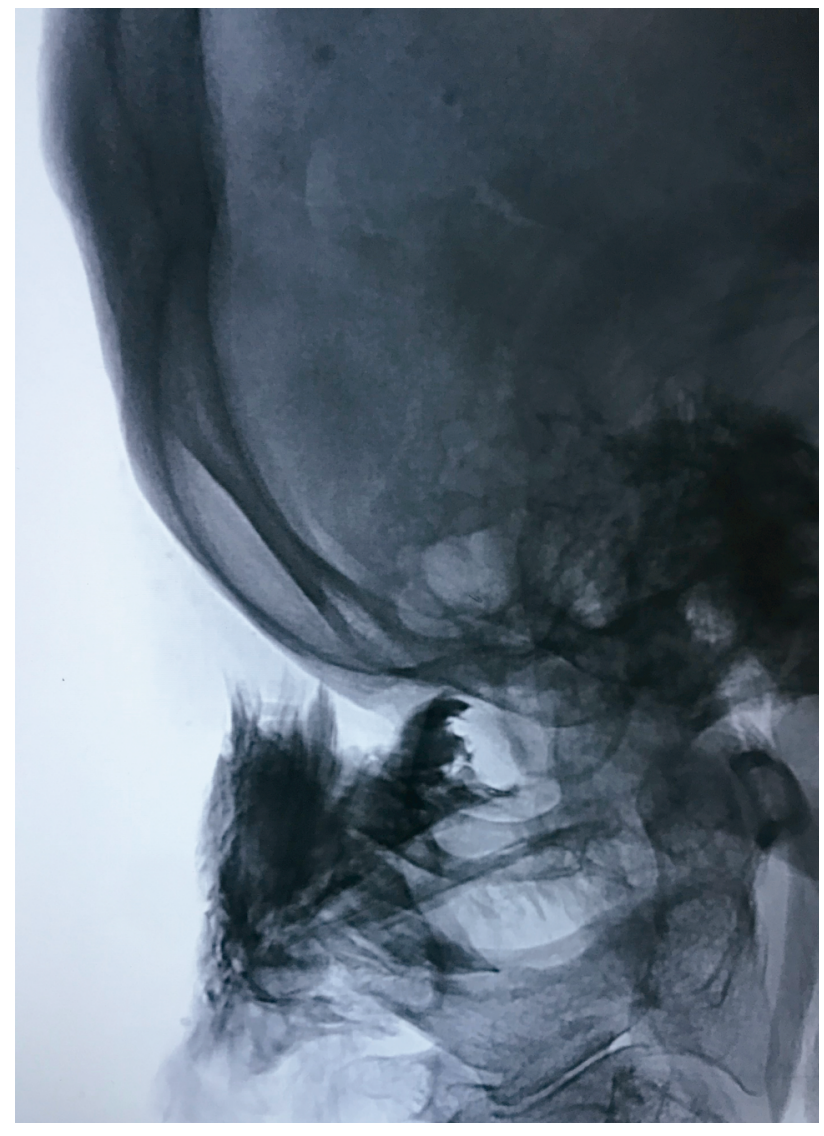

Fig. 2.

se aspiró para verificar punción inadvertida de vasos o duramadre. Una vez confirmado que no hubo salida de sangre o líquido cefalorraquídeo, se administraron $4 \mathrm{ml}$ de medio de contraste no iónico, observando una difusión hacia la región occipital de forma triangular (triángulo formado por los músculos oblicuo mayor, oblicuo menor, recto posterior de la cabeza y el semiespinoso de la cabeza) (Figura 2). Ya corroborado el objetivo terapéutico, se aspiró nuevamente y se administraron $5 \mathrm{ml}$ de bupivacaína (25 mg] con metilprednisolona no soluble $(40 \mathrm{mg}$ ) aforados en solución salina hasta un total de volumen de $10 \mathrm{ml}$ en el lado afectado, observando una adecuada disección de los planos musculares antes descritos en la imagen fluoroscópica. Se retiró aguja, se pasó a la paciente a recuperación sin incidentes, ni complicaciones.

Se evalúo la intensidad del dolor mediante la Escala Visual Análoga (EVA: sin dolor al máximo dolor) y la Escala Numérica (ENA: 0 = sin dolor, 10 = máximo dolor] antes del procedimiento, a las 2 horas, a las 24 horas, a los 30, 60 y 90 días posteriores al procedimiento. La paciente refirió mejoría de dolor a las 24 horas con una EVA = dolor leve y una ENA 2/10. Al seguimiento mensual hasta los 90 días, mantuvo los resultados satisfactorios $(E N A=2 / 10$ y EVA $=$ dolor leve), sin presencia de exacerbaciones o incremento al tratamiento farmacológico. 


\section{Caso 2}

Paciente femenina de 41 años, diseñadora, con antecedentes de síndrome de cirugía de espalda fallida lumbar portadora de equipo de estimulación medular con buen control referido a nivel lumbar. Se valora por dolor de tres meses de evolución a nivel cervical con irradiación occipital, unilateral derecha, de tipo punzante, paroxístico, continuo, con intensidad 8/10, EVA moderado, con exacerbación a los movimientos cervicales hasta 10/10, EVA severo. Con tratamiento a base de calor local, carbamazepina a dosis $200 \mathrm{mg}$ cada 24 horas, la cual se descontinuó por efectos intolerancia a los efectos adversos. Se decide realizar bloqueo diagnóstico del nervio occipital mayor en base a referencias anatómicas con $3 \mathrm{ml}$ de lidocaína al $2 \%$, refiriendo mejoría inmediata del $80 \%$ durante 2 horas, por lo que realizó una descompresión suboccipital percutánea sin incidentes. Refirió a las 24 horas posterior al procedimiento una EVA leve y una ENA 4/10, a los 30 días mantuvo una ENA 4/10 y EVA leve, a los 60 días refiere ENA 6/10 y EVA moderado y a los 90 días sostiene EVA moderado ENA 6/10. Sin presencia de crisis y sin tratamiento farmacológico.

\section{Caso 3}

Paciente femenina de 55 años, ama de casa, con antecedente de síndrome de cirugía de espalda fallida lumbar portadora de bomba intratecal con adecuado control a nivel lumbar. Refiere dolor de tres meses de evolución, en región cervical, unilateral izquierdo, de tipo punzante, ardoroso, de manera continua, con paroxismos intermitentes tipo "descarga eléctrica" sobre protuberancia occipital hacia dermatoma de C-2, con intensidad de acuerdo con el ENA 8/10 y al EVERA severo. Mejora parcialmente con el uso de calor local masajes, y el tratamiento médico por parte de su servicio tratante a base de paracetamol y ketorolaco. Se realiza bloqueo diagnóstico del nervio occipital mayor con lidocaína al $2 \%$, refiriendo una mejoría cercana al $100 \%$, posterior al procedimiento durante 24 horas, por lo que se programa para una descompresión suboccipital percutánea izquierda, la cual se realizó sin incidentes. A las 24 horas posterior al procedimiento, la paciente refirió una mejoría del dolor de acuerdo con la EVA ausente y una ENA 0/10, con una respuesta al alivio del dolor a los 90 días satisfactorios [EVA leve y ENA 2/10), sin presencia de crisis o tratamiento farmacológico indicado.

\section{Caso 4}

Paciente femenina de 69 años, con antecedentes de artritis reumatoide, coxartrosis bilateral bajo tratamiento con tramadol con paracetamol $37,5 \mathrm{mg} / 325 \mathrm{mg}$, con aparente buen control analgésico. Acude a consulta por referir dolor a nivel cervical unilateral derecho de tres meses de evolución, con irradiación a hombro derecho y región occipital ipsilateral de tipo opresivo, ardoroso, paroxístico, continuo, con una intensidad de acuerdo con la ENA 10/10 y la EVA severa. Se deci- de realizar bloqueo diagnóstico con anestésico local sobre el nervio occipital administrando $3 \mathrm{ml}$ de lidocaína al $2 \%$, observando una mejoría inmediata posterior al procedimiento durante 24 horas, por lo que se decide realizar una descompresión suboccipital percutánea derecha sin incidentes. Refirió a las 24 horas una mejoría del dolor con una EVA leve y una ENA 4/10. Al seguimiento mensual hacia los 90 días mantuvo la misma intensidad del dolor, sin presencia de crisis o tratamiento farmacológico.

\section{Caso 5}

Paciente femenina de 71 años con diagnósticos de carcinoma basocelular frontal, artritis reumatoide y coxartrosis bilateral bajo manejo médico a base de rituximab, tapentadol $50 \mathrm{mg}$ cada 12 horas, con regular control analgésico. Acude por dolor de 12 meses de evolución en región cervical izquierda con irradiación a hombro ipsilateral, punto gatillo sobre protuberancia occipital, refiriendo dolor de tipo punzante hacia opresivo, ENA 4/10, EVA leve ocasionalmente referido como moderado. Se manejó con infiltraciones miofasciales de puntos gatillo en región cervical con nula respuesta. Se realizó bloqueo del nervio occipital mayor en base a referencias anatómicas con lidocaína al 2 \%, refiriendo una mejoría cercana al $100 \%$ postprocedimiento durante 48 horas. Se programó para descompresión suboccipital percutánea, la cual se realizó sin incidentes, refiriendo a las 24 horas EVA ausente y ENA 1/10, manteniendo los mismos resultados referidos hacia los 90 días, donde comentó EVA leve y ENA 1/10, sin presencia de crisis o tratamiento farmacológico indicado.

\section{DISCUSIÓN}

La neuralgia occipital está considerada como una neuropatía frecuente. Esta fue descrita por Beruto y Lentijo y Ramos en 1821, siendo definida como una alteración incapacitante caracterizada por cefaleas recurrentes localizadas en la región occipital [13].

Actualmente existen diversas alternativas en el tratamiento médico incluyendo: a] la terapia conservadora utilizando medidas posturales y medios físicos, y b) la terapia farmacológica utilizando antidepresivos tricíclicos, inhibidores de la recaptura de serotonina, anticonvulsivantes, AINE, opioides o relajantes musculares, todos ellos con una baja eficacia demostrada y una cantidad representativa de efectos adversos en la mayoría de los pacientes (14).

El uso de los bloqueos de los nervios occipital mayor y menor, más que valor terapéutico sobre la enfermedad, posee valor diagnóstico y pronóstico, ya que poseen una duración y eficacia limitada. En un estudio realizado por Kuhn y cols. realizaron infiltración sobre el nervio occipital mayor utilizando esteroides y bupivacaína, obteniendo una respuesta positiva, refiriendo alivio total del dolor menos de una semana en el $10 \%$ de los pacientes, a la $1 .^{a}$ y $2 .^{a}$ semana el $30 \%$, y solo el $20 \%$ de los pacientes tuvieron una duración de 2,5 meses, compatible con los resultados obtenidos 
por Hanta y cols., realizando el mismo bloqueo y obteniendo una duración menor a una semana hasta en un $64 \%$ de los pacientes (15).

Otra alternativa de manejo intervencionista para este padecimiento, obteniendo resultados favorables en relación con el beneficio analgésico y el tiempo, es el estudio realizado por Justiz y cols., donde reportaron 29 pacientes con diagnóstico de neuralgia occipital a los que se les realizó un procedimiento de descompresión suboccipital. Se evaluó la intensidad del dolor de acuerdo con la EVA, el consumo de opioides y la funcionalidad en sus actividades diarias. Los resultados reportaron una disminución en la intensidad del dolor, con una EVA inmediatamente después del procedimiento de 7,9/10 a 1/10 (desviación estándar de 1,2]. El $58 \%$ de los pacientes refirieron una mejoría del dolor mayor al $50 \%$ a los 6 meses de seguimiento, y solo el $34 \%$ de los pacientes continuaban con mejoría al año del procedimiento. Hubo mejoría en la realización de sus actividades diarias y una disminución en el consumo de opioides hasta en un $56 \%$ de los pacientes [11].

En el estudio de Lauretti y cols. se incluyeron 30 pacientes, en los cuales comparó la infiltración directa en el nervio occipital mayor comparada con la descompresión suboccipital, llegando a la conclusión de que la duración de la inyección directa en el nervio tuvo una duración de 2 semanas en el alivio del dolor, en relación con la descompresión que tuvo una duración de 24 semanas, evaluando la calidad de vida en relación con el sueño, actividades diarias y concentración (15).

Los resultados en nuestros pacientes al realizar la descompresión suboccipital percutánea es que encontramos una mejoría significativa posterior al procedimiento con una duración mucho mayor a los bloqueos anestésicos locales sobre los nervios occipitales y con un beneficio analgésico mayor al uso de la terapia conservadora. No se presentaron efectos adversos y dichos resultados son similares a los reportes realizados por Justiz y Lauretti.

Sin embargo, también sabemos que se necesita una muestra mayor de pacientes para obtener mayor validez científica en nuestro medio, lo cual podría resultar difícil al ser una patología relativamente poco frecuente y mal diagnosticada.

Es conveniente decir que, a pesar de que la neuralgia occipital debe tratarse como cualquier caso de dolor neuropático, a nuestros pacientes se les propuso como primer plan de manejo el tratamiento farmacológico, se les explicaron beneficios y posibles efectos secundarios, pero no lo aceptaron, principalmente por los efectos secundarios, así que prefirieron el procedimiento intervencionista como primera opción. Ninguno de los pacientes presentaba dolor retrorbitario para pensar en la conexión de C-2 con el nervio trigémino, por lo que se descartaron casos de migraña o cefalea en racimos como diagnósticos diferenciales.

Aunque las complicaciones de la técnica son poco frecuentes por el uso de una aguja con punta cortante, es necesario en todo momento la visualización de la punta de la aguja, sobre todo en áreas de alta vascularidad o inervación, para evitar una punción vascular, intraneural o de duramadre, evitando así un paro car- diorrespiratorio, infarto del tronco cerebral, cuadriparesia o convulsiones (12).

Cabe mencionar intentar la realización del procedimiento con agujas de bisel corto o romas, aunque la aspiración negativa no garantiza la ausencia de punción vascular, como lo menciona Candido y cols. en su reporte (16).

Las relaciones del nervio occipital mayor con el ramo dorsal de C-2, con numerosos músculos: oblicuo inferior de la cabeza, recto posterior mayor y menor, entre otros, además con una vascularidad importante, hacen que el uso de la ultrasonografía se deba tomar en cuenta para la realización de este procedimiento, mejorando la seguridad y eficacia, así como la disminución de las posibles complicaciones [17,18].

\section{CONCLUSIONES}

Consideramos que el uso de la descompresión percutánea del nervio occipital mayor vía suboccipital puede ser tomada como una alternativa en pacientes con neuralgia occipital, con una alta probabilidad de obtener un beneficio analgésico y una baja probabilidad de presentar algún efecto adverso o complicación.

\section{CONFLICTO DE INTERESES}

Los autores declaran no tener ningún conflicto de intereses.

\section{BIBLIOGRAFÍA}

1. Headache Classification Subcommittee of the International Headache Society. The International Classification of Headache Disorders. 2nd ed. Cephalalgia. 2004;24(Suppl 1): 9-160. DOI: 10.1111/j.1468-2982.2003.00824.x.

2. Hammond SR, Danta G. Occipital neuralgia. Clin Exp Neurol. 1978;15:258-70.

3. Cornely C, Fischer M, Ingianni G, Isenmann S. Greater occipital nerve neuralgia caused by pathological arterial contact: treatment by surgical decompression. Headache. 2011;51(4):609-12. DOI: 10.1111/j.15264610.2010.01802.x.

4. Loukas M, El-Sedfy A, Tubbs RS, Lois RG, Wartmann $\mathrm{CH}$, Curry B, et al. Identification of greater occipital nerve landmarks for the treatment of occipital neuralgia. Folia Morphol (Warsz). 2006;65(4):337-42.

5. Dougherty C. Occipital Neuralgia. Curr Pain Headache Rep. 2014;18(5):411. DOl: 10.1007/s11916-014-0411-x.

6. Longo G, Osikowicz M, Ribeiro-da-Silva A. Sympathetic fiber sprouting in inflamed joints and adjacent skin contributes to pain-related behavior in arthritis. J Neurosci. 2013;33(24):10066-74. DOI: 10.1523/JNEUROSCl.5784-12.2013.

7. Anthony M. Headache and the greater occipital nerve. Clin Neurol Neurosurg. 1992;94(4):297-301.

8. Patwardhan AG, Khayatzadeh S, Havey RM, Voronov LI, Smith ZA, Kalmanson O, et al. Cervical sagittal balance: a biomechanical perspective can help clinical practice. Eur Spine J. 2018; 27(Suppl 1):25-38. DOI: 10.1007/s00586017-5367-1. 
9. Güvencer M, Akyer P, Sayhan S, Tetik S. The importance of the greater occipital and the suboccipital región for nerve blockade and surgical approaches. An anatomic study on cadavers. Clin Neurol Neurosurg. 2011;113(4):289-94. DOI: 10.1016/j.clineuro.2010.11.021.

10. Trescot A, Racz G, Nagy L. Suboccipital decompression for occipital neuralgia. J Spine Neuroscience. 2017;1(1):4-11.

11. Justiz R, Taylor V, Racz G B, Meta G. Suboccipital decompression: A retrospective analysis of a novel technique for the treatment of occipital neuralgia. Fifth World Congress of the World Institute of Pain abstracts; March 2009; New York, NY.

12. Racz GB, Noe CE, Justiz R. A new technique for suboccipital compartment decompression. Pain Pract. 2009;9(51):29. DOI: 10.1111/j.1533-2500-2009-00266.x.

13. Perelson HN. Occipital nerve tenderness; a sign of headache. South Med J. 1947;40(8):653-6. DOI: 10.1097/00007611-194708000-00005.
14. Martelletti P, van Suijlekom H. Cervicogenic headache: practical approaches to therapy. CNS Drugs. 2004;18(12):793805. DOl: 10.2165/00023210-200418120-00004.

15. Lauretti GR, Correa SW, Mattos AL. Efficacy of the Greater Occipital Nerve Block for Cervicogenic Headache: comparasing classical and subcompartmental technique. Pain Pract. 2015;15(7):654-61. DOI: 10.1111/papr.12228.

16. Candido KD, Ghaly RF, Mackerley S, Knezevic NN. A Whitacre-type spinal needle does not prevent intravascular injection during cervical nerve root injections. South Med J. 2010;103(7):679-82. DOI: 10.1097/ SMJ.Ob013e3181e1e3d6.

17. Nauroze S. Occipital Neuralgia Diagnosis and Treatment: The Role of Ultrasound. Headache. 2016;56(4):801-7. DOI: 10.1111/head.12790.

18. Chang KV, Wu WT, Oscakar L. Ultrasound-Guided Interventions of the Cervical Spine and Nerves. Phys Med Rehabil Clin N Am. 2018;29(1):93-103. D0I: 10.1016/j.pmr.2017.08.008 